\title{
El Efecto Educación en el Desarrollo Social: Intelectual y Políticamente Subestimado ${ }^{1}$
}

\section{David P. Baker}

\section{Universidad Estatal de Pennsylvania}

David P. Baker es profesor de Educación Comparada y Sociología en la Universidad Estatal de Pennsylvania, donde dirige un programa de investigación sobre el impacto de la globalización de la educación y las tendencias internacionales. Su investigación en Ciencias Sociales incluye 60 países. Actualmente dirige un proyecto financiado por la National Science Foundation, proyecto de investigación multidisciplinario orientado a comprender los efectos de la educación en la salud de la población, incluyendo el HIV así como la pandemia del SIDA en África Subsahariana y la salud en las zonas alto andinas del Perú.

Es consultor frecuente en temas de desarrollo social, económico y desarrollo educativo para varias agencias multilaterales como la OECD, el Banco Mundial y UNESCO.

1 Artículo presentado originalmente como discurso para el 30 aniversario del Grupo de Análisis para el Desarrollo (GRADE), Lima, Perú, el 10 de noviembre de 2010. El autor agradece a Santiago Cueto por sus útiles comentarios sobre un primer borrador. 


\title{
El Efecto Educación en el Desarrollo Social: Intelectual y Políticamente Subestimado
}

\begin{abstract}
Resumen
La educación básica y avanzada está ahora expandiéndose rápidamente en todo el mundo, y la literatura de investigación demográfica y epidemiológica está pletórica de resultados que muestran la sólida asociación positiva entre nivel de educación y salud, además de otros comportamientos y actitudes individuales esenciales para el desarrollo social. Este "efecto educación" es tan pronunciado que cabe suponer que la educación es una de las principales causas de la primera transición demográfica de la sociedad moderna, consistente en índices más bajos de mortalidad y fecundidad y mayor esperanza de vida, que indican una mejora significativa en la salud y desarrollo de la población en general. La primera transición demográfica ya ha ocurrido en muchas naciones en todo el mundo pero, para las naciones más pobres, esta transición sigue siendo un desafío crucial para el desarrollo social sostenible. Aunque la mayoría de demógrafos e investigadores de la salud reconocen la significativa y persistente asociación entre escolaridad formal y resultados positivos en la salud, la razón de que la educación tenga esta influencia no es bien comprendida. Habiendo de por medio cruciales implicaciones de política, existe confusión sobre el papel de la educación en las políticas de desarrollo social.
\end{abstract}

Palabras clave: educación y desarrollo social, educación y transición demográfica, efecto educación, expansión de la educación 


\title{
Education Effect on Social Development: Intellectually and Politically Underestimated
}

\begin{abstract}
Basic and advanced education is now rapidly expanding around the world, and demographic and epidemiological research literatures brim with results showing the robust positive association between educational attainment and health, plus other individual behaviors and attitudes essential for social development. This "education effect" is so pronounced that education can be assumed to be a major cause of the first demographic transition of modern society, consisting of reduced mortality and fertility, and longer life spans, which indicate a significant improvement in overall population health and development. The first demographic transition has already occurred in many nations worldwide, but for poorer nations this transition remains a crucial challenge to sustainable social development. While most demographers and health researchers acknowledge the persistent and significant association between formal schooling and positive health outcomes, why education has this influence is not well understood. With crucial policy implications hanging in the balance, there is confusion over the role of education in social development policy.
\end{abstract}

Keywords: education and social development, education and demographic transition, effect education, expansion of education 
E tre el público, la educación formal es ensalzada irrealistamente como una panacea para todos los problemas sociales. Sin embargo, entre los intelectuales y los asesores de política, la educación es subestimada como una fuerza causal en la sociedad moderna. La ingenua sobrestimación entre el público del potencial de la educación para la sociedad alimenta, sin duda, la visión limitada de los intelectuales y asesores de política. Al mismo tiempo, una extensa literatura de investigación que atraviesa muchas disciplinas académicas informa de numerosas asociaciones entre los niveles de educación y todo tipo de actitudes y comportamientos individuales. Por ejemplo, un reciente meta-análisis del efecto educación sobre la mortalidad en todas sus causas en los países desarrollados y en desarrollo encuentra consistente y fuerte evidencia de que ceteris paribus más educación lleva a una vida más larga (Baker, León, Smith, Collins y Movit, 2011). La extensa literatura de investigación sobre los efectos de la educación en la salud y el desarrollo social, junto con el efecto causal subestimado de la educación, dan lugar a un discurso intelectual paradójico sobre la educación y el desarrollo social. Aunque se conoce que la educación está ampliamente asociada con muchas actitudes y comportamientos importantes para el desarrollo social, las ideas comunes sobre la influencia causal de la educación son limitadas y han sido puestas a prueba solo débilmente.

A la luz de nueva teoría e investigación, se propone aquí una solución a la paradoja del efecto educación. Recientes hallazgos empíricos, valorados desde una nueva perspectiva, sobre la relación causal dinámica entre educación y sociedad, indican que la educación tiene un impacto mucho mayor en el desarrollo social, económico y de la salud que lo que se reconoce en la comunidad de desarrollo. Aquí se esboza especialmente una nueva evaluación del efecto que la educación opera en la sociedad que lleva a una teoría explícitamente cognitiva del impacto causal de la educación formal sobre las actitudes y el comportamiento. A esto le sigue una breve descripción de recientes investigaciones peruanas y africanas sobre el efecto educación en la salud de la población, que demuestra el impacto causal potencialmente importante de la educación en las habilidades metacognitivas. Por último, se consideran las propiedades cognitivas transformadoras de la revolución educativa junto con el creciente papel dominante de la educación en la consecución de estatus social como una explicación para la intensificación de la cultura educativa y su influencia en el desarrollo social en la sociedad posindustrial. 


\section{La Demografía de la Revolución Educativa}

Un breve panorama de la demografía de la expansión educativa proporciona el contenido societal general de la paradoja efecto educación-desarrollo social. La educación formal ha crecido enormemente en todo el mundo; el crecimiento de la educación de masas, un fenómeno social relativamente nuevo durante el siglo XX, amplió la matrícula en la escuela primaria, secundaria y terciaria. En un sentido demográfico, ir a la escuela durante un número considerable de años es un cambio nuevo y de gran escala en el comportamiento de niños y jóvenes, así como de sus familias y comunidades, sin paralelo en la sociedad humana premoderna, tradicional. En función de la institucionalización intensiva de la educación formal, el $80 \%$ de todos los seres humanos de 15 años o más saben leer y escribir una breve declaración sobre su vida. La mayoría de las personas que todavía son analfabetas viven en las naciones pobres y, de ellas, 60-70\% son mujeres (UNESCO, 2002).

Esta expansión en el acceso a la escolaridad, a menudo denominada revolución educativa, ha transformado la sociedad postindustrial. Junto con unos cuantos otros fenómenos importantes como el capitalismo global y la democracia representativa, la escolaridad de poblaciones enteras durante cada vez más años para completar una variedad creciente de grados educacionales cambia tanto a los individuos como a muchas otras instituciones que componen el núcleo de la sociedad. La revolución educativa es un fenómeno cultural que tiene importantes consecuencias materiales y políticas. La educación generalizada en la sociedad posindustrial crea ideas culturales sobre nuevos tipos de conocimientos, nuevos tipos de expertos, nuevas definiciones de éxito y fracaso personal, un nuevo lugar de trabajo y concepción de los puestos de trabajo, nuevas definiciones de la inteligencia y el talento humano, y mucho más (Baker, s. f. ). Al mismo tiempo, el nivel de educación y la obtención de grados académicos han llegado a dominar la estratificación social y la movilidad social, poniendo de lado y deslegitimando los medios pasados para la consecución de estatus (Hout, 1988; Torche, 2010). El impacto global de la educación formal en la sociedad posindustrial ha sido tan extenso que la educación de las masas es una revolución social fundadora de la modernidad (Parsons, 1971). 


\section{Débil Teoría Sobre la Educación y la Sociedad}

Aunque pueden formularse argumentos convincentes acerca de que la revolución educativa ha cambiado muchas instituciones sociales durante el último siglo, generalmente la educación es vista entre intelectuales y analistas de política social como lo que puede llamarse una institución secundaria. Esto significa que la escolaridad - educar a los estudiantes a través de la enseñanza y un plan de estudios - desempeña principalmente un "papel de ayuda" en la creación de la complejidad social y económica general de la sociedad, y de allí la noción de la educación como un reproductor de la sociedad entre cada generación sucesiva de niños. Esta concepción del papel de la educación en la sociedad es tan común y tan carente de oposición, que teorías tan diversas entre sí como el marxismo y la teoría del capital humano asumen esencialmente este modelo como cierto, aunque con consecuencias diametralmente opuestas para la sociedad.

Una expectativa de solo limitados efectos de la escolaridad sobre los individuos más una falta general de consideración de los posibles efectos macro institucionales son la causa principal de la paradoja efecto educación en general y de la paradoja efecto educación-desarrollo social en particular (Baker et al., 2011). Asumir que la educación es una institución secundaria deja a la teoría social y a la estrategia de políticas sin preparación para incorporar la plena implicación del inmenso número de asociaciones empíricas reportadas entre nivel de educación y comportamiento centrales para la salud, el comportamiento económico y otros aspectos del desarrollo social. En cambio, pese a esta literatura, el lamentable y demasiado común resultado de política es trivializar el efecto educación como solo un indicador de la "fuerza causal real" de la clase social (por ejemplo, poder y recursos materiales), o sugerir que la escolaridad genera principalmente resultados no educativos tales como postergación de la gratificación y autoeficacia. Si bien es cierto que el nivel de educación domina cada vez más la consecución del estatus social y que los individuos que están expuestos a la educación formal sí tienden a desarrollar mayor autoeficacia y otros rasgos psicológicos, todo esto probablemente no explica la gran mayoría de los efectos de la educación. Lo que se necesita es un mejor modelo de qué papel desempeña la educación en la sociedad humana, dados los casi cien años de la revolución educativa. 


\section{La Teoría Neoinstitucional de la Educación como Institución Primaria}

La teoría neoinstitucional pone de cabeza la visión tradicional de la relación entre educación y sociedad -la sociedad sigue a la educación más que al revés - y, por lo tanto, la educación formal de masas es una fuerza constructiva fundamental en la sociedad. Debido al impacto histórico de la revolución educativa, la educación se ha convertido en una institución primaria que da forma a muchas otras instituciones sociales (Baker, s. f.; Parsons, 1971). Pueden formularse sólidos argumentos de que la educación, como se ha practicado durante el pasado siglo y medio, es mucho más que un ejercicio preparatorio para los jóvenes que solo va por donde se lo dictan las demandas tecnológicas y sociales de la sociedad. Por el contrario, la revolución educativa ha construido, para bien o para mal, muchas de las ideas, creencias y capacidades humanas básicas en que se basa la sociedad humana a principios del siglo XXI. Por ejemplo, ideas tales como valores y conocimientos universalistas, empoderamiento humano, justicia social, ciudadanía, pretensiones de verdad científica, meritocracia y racionalidad han impregnado completamente la cultura moderna, en gran parte, gracias al éxito de la educación formal como institución social, desde la educación en la primera niñez hasta los estudios de posgrado en las universidades. En la sociedad escolarizada, no solo todos son estudiantes a quienes se considera capaces de un aprendizaje académico que los transformará para poseer competencias importantes, sino que también la sociedad se transforma en el proceso de educar a todos (Meyer, 1977).

La sociedad escolarizada tiene implicaciones de mucho más alcance para la vida humana, algunas de las cuales son consideradas como positivas y, otras, como desconcertantes. Más allá de documentar el considerable impacto de la educación sobre el individuo, una cantidad sustancial de investigaciones encuentra evidencia del impacto institucional de la revolución educativa sobre las ideas, valores y normas de otras instituciones, tales como: trabajo y ocupaciones (Baker, 2009), funciones de los padres y comportamiento normativo (Schaub, 2010), estructura y procesos del sistema de gobierno y cultura cívica en las sociedades democráticas (Kamens, 2009), definiciones de conocimiento y pretensiones de verdad (Kamens, Meyer y Benavot, 1996; Young, 2008), valoración de las capacidades humanas centrales (Martínez, 2000; Blair, Gamson, Thorne y Baker, 2005), organización de las comunidades religiosas y teología (Schwadel, 2003), definiciones de éxito y fracaso personal 80 (Smith, 2003), propagación y dominio de las organizaciones formales 
(Stinchcombe y March 1965), creciente creencia en el profesionalismo y profesionalidad, y cientización de la sociedad (Drori, Meyer y Hwang, 2006), y la imagen fundacional de la propia sociedad (Frank y Gabler, 2006).

Desde principios de la década de 1990 se había planteado una serie de ideas sobre un mecanismo causal directo existente tras el efecto educación sobre la salud y otras áreas del desarrollo social, aunque pocas han sido plenamente desarrolladas o puestas a prueba rigurosamente. Las especulaciones sobre los mecanismos causales existentes tras el efecto educación son generalmente de dos tipos. Uno se centra en una visión literal pero limitada de la educación, que sugiere que la escolaridad proporciona información básica sobre la salud con alguna preparación para realizar tareas como leer instrucciones médicas y seguir indicaciones (Cleland y Van Ginneken, 1988). El segundo tipo de especulación sugiere que la educación formal, de alguna manera, infunde nuevas actitudes que conducen a una mejor salud y longevidad (véase una reseña en Hobcraft, 1993). Estas actitudes incluyen cualidades psicológicas individuales, como postergación de la gratificación, cambios en los patrones interactivos dentro de las familias y una actitud más fuerte de empoderamiento personal. Aunque el supuesto detrás de cada tipo de especulación no ha sido probado extensamente, la educación formal probablemente transforma a los individuos de muchas maneras, y estas ideas sobre el mecanismo causal de la escolaridad en la salud pueden ser parte de la respuesta. Sin embargo, estos dos tipos de argumentos causales asumen principalmente el proceso de escolaridad como una caja negra, en que ninguno aborda las actividades principales del aprendizaje. Interpretaciones más literales de la transferencia de información a través de la alfabetización asoman en el cuadro pero no van más allá, y las ideas sobre el cambio de actitudes se saltan sobre los procesos de aprendizaje primario en su conjunto.

En esencia, la escolaridad es un entorno único en el que los estudiantes pasan largos periodos dedicados a actividades cognitivas, y este proceso es la clave para comprender el mecanismo causal detrás del efecto educación sobre la salud. Aprender a leer, escribir en un idioma y utilizar números, incluso en condiciones rudimentarias, no son solo habilidades específicas, porque cada una es resultado de un considerable ejercicio cognitivo abstracto que probablemente transforma cómo los individuos escolarizados piensan, razonan y resuelven problemas (por ejemplo, Martínez, 2000). Además, hay evidencia de que la escolaridad se vuelve progresivamente más cognitiva en sus exigencias a medida que los estudiantes pasan a grados superiores. 
Y es esta mejora metacognitiva progresiva derivada de la escolaridad lo que puede resultar ser el mecanismo más eficaz para crear el efecto educación sobre la salud; nueva investigación demuestra convincentemente que la escuela formal es una institución de desarrollo neurocognitivo (Baker, Salinas y Eslinger, s. f.). Y, a partir de evidencia, desde principios de la expansión de la educación en las naciones desarrolladas y ahora de las naciones en desarrollo, es claro que incluso pequeñas dosis de escolaridad pueden crear diferencias con la socialización no formal tradicional de los niños no escolarizados. La educación formal sume a los individuos en un proceso cognitivo singularmente diferente en comparación con el relacionado con la agricultura de subsistencia, el trabajo fabril de principios de la era industrial y otras actividades premodernas. Con la excepción de las creencias sagradas y mágicas, antropológicamente hablando, durante la mayor parte del curso de la sociedad humana la gran masa de la gente no perteneciente a la élite vivía en un mundo muy concreto.

El advenimiento relativamente reciente de la educación de masas es la más grande intervención individual no familiar para los niños, y una parte considerablemente grande de su proceso central es una intervención cognitiva. Un posible efecto metacognitivo robusto de esta intervención cognitiva en el desarrollo social puede haber pasado desapercibido en gran medida desde que la revolución educativa hace la escolaridad tan ubicua en tantos lugares. Sin embargo, hay evidencia reciente de que la revolución educativa ha extendido en todo el mundo una versión relativamente similar a la escolaridad occidental, que incluye importantes posibilidades para el cambio cognitivo en los individuos (Baker y LeTendre, 2005). Por lo tanto, una hipótesis cognitiva es una vía prometedora para teorizar sobre cómo funciona el efecto educación sobre la salud y el desarrollo social de los individuos.

\section{Hipótesis Escolaridad-Cognición-Desarrollo Social}

Una de las principales causas de la paradoja educación-desarrollo social es la falta de una teoría de un efecto de la escolaridad sobre cómo la exposición a la escolaridad (aparte del prestigio material y social acumulado a partir del nivel de educación) influye en la salud individual y en los resultados sociales. Sin tal teoría, la subestimación del efecto educación probablemente continuará y debilitará los argumentos a favor del gasto público en la expansión de las escuelas para todos los niños y jóvenes. 
Un mecanismo causal plausible detrás del efecto educación sobre los individuos es que la escolaridad, debido al aprendizaje de habilidades académicas básicas tales como lectura, escritura y cálculo, acrecienta las habilidades cognitivas de orden superior (tales como razonamiento, resolución de problemas novedosos, pensamiento esforzado y planificación de tareas) que, a su vez, ayudan a los individuos a transformar datos básicos en conocimientos más profundos que mejoran las habilidades de evaluación de riesgos y toma de decisiones y comportamientos relacionados con la salud y otras cuestiones del desarrollo social, como las decisiones económicas. Lo que puede llamarse hipótesis escolaridad-cognición-desarrollo social propone que: a través de la enseñanza de la lectura, la escritura, el cálculo y otros temas académicos, la escolaridad mejora las habilidades cognitivas de orden superior (tales como razonamiento, resolución de problemas novedosos, pensamiento esforzado y planificación de tareas), que a su vez ayudan a los individuos a transformar datos básicos en conocimientos más profundos que mejoran las habilidades de evaluación de riesgos y toma de decisiones sobre la salud y otras decisiones cruciales de la vida.

Esta hipótesis tiene la ventaja de que se enfoca en la tarea central de la escolaridad - el desarrollo de habilidades cognitivas-, un proceso que ha sido subestimado en la investigación en educación en materia de salud y desarrollo social. La hipótesis tiene la ventaja adicional de ser compatible con nuevas conclusiones centrales sobre educación y desarrollo cognitivo, como recientes investigaciones sobre el efecto de la escolaridad en el desarrollo neurológico, que establecen tres conclusiones interrelacionadas que respaldan esta hipótesis.

En primer lugar, el desarrollo neurológico de habilidades cognitivas de orden superior se produce al menos hasta la adolescencia tardía y es altamente sensible a la estimulación ambiental, como la que ocurre rutinariamente en la educación formal. Estudios de trastornos neurológicos y psiquiátricos infantiles y de niños normalmente desarrollados sin ningún trastorno clínico muestran que las habilidades cognitivas de orden superior se producen hasta la adolescencia tardía y constituyen un conjunto específico de capacidades cognitivas (por ejemplo, Blair, 2006; Duncan, Burgess y Emslie 1995; Eslinger, Flaherty-Craig y Benton, 2004; Shallice y Burgess, 1991). Además, una serie de experimentos con fMRI (functional magnetic resonance imaging o resonancia magnética funcional) sobre desarrollo del cerebro encuentran que cuando niños en edad escolar resuelven nuevos problemas matemáticos similares a los utilizados comúnmente en los planes 
de estudio de matemáticas, se activan las áreas cerebrales asociadas con habilidades cognitivas de orden superior (a saber, activaciones reclutadas en la corteza parietal superior más prominentemente, las cortezas dorsolateral prefrontal, occipital-temporal y premotora/suplementaria, los ganglios basales y la ínsula) (Eslinger, Blair, Wang, Lipovsky, Realmuto, Baker y Yang, 2009).

En segundo lugar, la exposición a la educación formal está monotónica y linealmente asociada con mayores habilidades cognitivas de orden superior. Un meta-análisis de más de 50 estudios utilizando observación naturalista, comparaciones estadísticas post hoc y análisis secuenciales de cohorte concluye que, por cada año de asistencia a la escuela, descontando factores socioeconómicos, existe un aumento monotónico en las habilidades cognitivas relacionadas con el $\mathrm{Cl}$ (Ceci, 1991). También, estudios cuasi experimentales de adultos no escolarizados y escolarizados en comunidades de agricultura de subsistencia encuentran que pequeñas dosis de escolaridad en la niñez producen habilidades cognitivas de orden superior entre adultos, sin considerar condición social y económica, y condiciones de trabajo (por ejemplo, Christian, Bachman y Morrison, 2001; Cole, 1996; Luria, 1976; Stevenson, Parker, Wilkinson, Bonnevaux, Gonzalez y Greenfield, 1978; Stevenson, Chen y Booth, 1990; para un examen completo véase Baker et al., s. f.).

En tercer lugar, las habilidades cognitivas de orden superior expresadas como mejor habilidad para el cálculo están asociadas con mejores habilidades para evaluación de riesgos y toma de decisiones. El cálculo se aprende principalmente en la educación formal, y experimentos sobre evaluación de riesgos y el uso de la heurística efectiva para la toma de decisiones encuentran repetidamente que tales habilidades están relacionadas positivamente con el cálculo y las habilidades cognitivas de orden superior (Bruine de Bruin, Parker y Fischhoff, 2007; Peters, Västfjäll, Slovic, Mertz, Mazzocco y Dickert, 2006). Aunque esta hipótesis ha sido desarrollada y sometida a prueba para comportamientos y decisiones sobre la salud, puede generalizarse a otros ámbitos del desarrollo social.

\section{Investigación Peruana y Africana sobre la Hipótesis}

La hipótesis escolaridad-cognición-desarrollo social abre una serie de 84 maneras de investigar el efecto educación. Una poderosa manera es comparar 
individuos con grados significativamente diferentes de escolaridad en una variedad de habilidades cognitivas. En las sociedades más profundamente escolarizadas de América del Norte y Europa occidental, incluso el nivel más bajo de escolaridad dura un número considerable de años, y además no hay mucha variación en la educación entre las poblaciones. En las naciones en desarrollo, la situación es diferente. Es posible encontrar una gran variedad en la escolaridad entre adultos con funciones normales, incluyendo un número significativo de adultos no escolarizados o con baja escolaridad. Además, hay regiones donde adultos con grados variables de escolaridad están relativamente aislados y tienen limitado contacto con medios de comunicación y ocupaciones que podrían ampliar sus habilidades cognitivas. Los Andes del Perú son uno de esos lugares.

Con colaboradores peruanos, una muestra de alrededor de 300 adultos quechuahablantes con dosis variables de escolaridad, incluyendo no escolarizados, recibieron una extensa serie de pruebas cognitivas, incluida la evaluación de inteligencia más cristalizada más el tipo de habilidades cognitivas que, según la hipótesis, son mejoradas por la escolaridad². La modelación de estos datos muestra que, después de ajustar una variedad de factores demográficos y nivel de inteligencia cristalizada, incluso pequeñas dosis de escolaridad formal mejoraron las habilidades cognitivas de orden superior de inteligencia fluida junto con razonamiento, resolución de problemas novedosos, pensamiento esforzado y planificación de tareas (Baker et al., s. f.). Se encontró que los efectos de la selección en términos de quiénes fueron a la escuela estaban solo mínimamente relacionados con el nivel de inteligencia del sujeto estimado por la PPVT (Peabody Picture Vocabulary Test), y los resultados básicos se mantienen después de que se añadieron diversas pruebas y ajustes al efecto de selección.

Los datos peruanos ponen a prueba fuertemente la primera mitad de la hipótesis que predice que, durante el aprendizaje de la lectura, la escritura y el cálculo, incluso en solo unos pocos años de escolaridad primaria, las habilidades cognitivas de ámbito general (por ejemplo, memoria operativa y capacidad de razonamiento abstracto), las habilidades para la toma de decisiones arriesgadas que proporcionan los medios necesarios para apreciar las consecuencias de los riesgos, y la capacidad de razonar adecuadamente en situaciones novedosas también se ven mejoradas. Examinar la influencia

2 Martín Benavides y colegas en GRADE, Lima, Perú. 
de estas habilidades cognitivas mejoradas por la escolaridad sobre la salud y otros comportamientos relacionados con el desarrollo social pone a prueba la segunda mitad de la hipótesis escolaridad-cognición-desarrollo social.

Con colaboradores ghaneses y otros, datos similares recopilados entre 181 adultos no escolarizados y escolarizados en el sector rural de Ghana incluyeron también los conocimientos del sujeto respecto de la infección del VIH/SIDA y sus comportamientos para reducir el riesgo, incluyendo asistir a reuniones informativas y clases sobre VIH, comunicación sobre el VIH y su prevención con una pareja sexual, detección y conciencia de la condición seropositiva propia y de la pareja, y uso del preservativo ${ }^{3}$. Como en muchos otros países africanos subsaharianos, la pandemia del VIH/ SIDA es una de las principales amenazas para la salud de la mayoría de los individuos en Ghana. Utilizando el modelado de ecuaciones estructurales, el análisis encuentra que las capacidades cognitivas, el dominio del cálculo y las habilidades para la toma de decisiones aumentaron con la exposición a la escolaridad tal como en los datos peruanos, pero, aun más, estas habilidades mejoradas tuvieron parte en los efectos de la educación sobre los comportamientos protectores. En otras palabras, en promedio, los individuos con habilidades cognitivas mejoradas por la escuela fueron más propensos a comprender con mayor precisión sus riesgos y a asumir comportamientos y estrategias de prevención (Peters, Baker, Dieckmann, León y Collins, 2010).

Compensando con el detalle de las mediciones la capacidad para generalizar, tanto las muestras peruanas como las ghanesas fueron recogidas en una sola región, y abarcaron relativamente pocos sujetos que fueron sometidos a una amplia medición de habilidades cognitivas. Para estimar el grado en que la hipótesis escolaridad-cognición-desarrollo social se generaliza a una población completa, se llevó a cabo un tercer estudio (Baker, León y Collins, 2010). El análisis de grandes muestras representativas a escala nacional de Encuestas Demográficas y de Salud (EDS) de nueve países del África subsahariana recopiladas entre 2003 y 2005 encuentra que, descontando las variables de control, hay una robusta influencia positiva de la educación sobre el uso del preservativo entre adultos sexualmente arriesgados. Y lo que es importante, la educación influye robustamente en la capacidad para razonar sobre la salud, y este factor interviene en una proporción significativa del efecto educación sobre el uso del preservativo. 
Estas son solo tres pruebas de la hipótesis escolaridad-cognición, y solo se aplican a resultados en materia de salud, pero la evidencia apoya el argumento general. Aunque esta hipótesis es un comienzo para entender la asociación de largo alcance entre educación y desarrollo social, no explica por sí misma por qué la educación formal continúa avanzando en todas partes en la cultura posindustrial. Si bien existe considerable evidencia sobre los efectos de la escolaridad como institución primaria sobre los individuos y una variedad de procesos institucionales de la sociedad, identificar tales efectos no explica por qué la educación ha llegado a tener el significativo poder transformador que aparentemente tiene. Esto requiere examinar dos funciones dinámicas de la educación en la sociedad.

\section{Funciones Sociales de la Educación que se Refuerzan Mutuamente}

John Meyer, en su artículo seminal de 1977 (Meyer, 1977) sobre el poder transformador de la revolución educativa "Los efectos de la educación como institución", planteó la hipótesis de que la educación tiene dos funciones societales emergentes que se refuerzan mutuamente y que, por tanto, generarán un significativo poder institucional. Una es la creciente función distributiva de la educación y la otra es su también creciente función cognitiva ${ }^{4}$.

Distribución es una manera corta de expresar el papel que la educación formal desempeña ahora en la consecución de estatus social, que incluye principalmente, pero no solamente, el nivel ocupacional. Cognición significa aquí el considerable cambio en los procesos de pensamiento que se produce entre individuos escolarizados, que abarca al menos tres facetas principales: 1) aprendizaje del plan de estudios manifiesto, 2) desarrollo de razonamiento más profundo, resolución de problemas y pensamiento racional, y 3) expansión de las imágenes conceptuales de uno mismo y del mundo. Específicamente, la hipótesis de Meyer predice que con el tiempo y a través de cada vez más individuos, las funciones distributiva y cognitiva de la escolaridad se reforzarán entre sí y formarán una ideología significativa sobre la centralidad de la educación formal, lo cual a su vez intensificará la

4 Cognitivo se usa aquí en lugar de la formulación original de Meyer en 1977 de socialización, pues el primer término representa con más precisión lo que hace la escolaridad; además, el segundo es un término más antiguo ahora rara vez usado y transmite otros significados innecesarios. 
legitimidad de cada función. Además, Meyer planteó que este proceso simbiótico presta a la educación formal la capacidad cultural de cambiar no solo a los individuos, sino, y quizá lo más relevante para la sociología, también a otras instituciones sociales.

La investigación que se acaba de reseñar sobre el efecto cognitivo de la educación en la salud es un buen ejemplo de la función cognitiva. La función distributiva, si bien es conocida en la sociología, puede ser menos apreciada por los investigadores de la educación como función social dominante.

Desde el inicio de la revolución educativa en el siglo XIX, ha habido una constante tendencia hacia el predominio de la educación en la consecución de estatus social. En los últimos tiempos esto se ha acelerado hasta el punto de que incluso el efecto del estatus de la educación de los padres palidece en comparación con la propia educación del individuo. Por ejemplo, el histórico estudio estadounidense de Hout de 1988 (Hout, 1988; véase también Hout, 1984) encuentra que, a fines de la década de 1980, había todavía influencia del origen social de los padres en el nivel de educación de los hijos, pero la fuerza de esta relación había disminuido en todo un tercio de lo que había sido en la década de 1960, y entre la descendencia que concluía una carrera universitaria la relación estaba completamente desconectada. Esto significa que, por primera vez, había evidencia de que la distribución basada en el nivel de educación del individuo independiente del origen social se había convertido en el proceso dominante de la consecución de estatus.

En un estudio integral sobre la consecución de estatus social en Estados Unidos, Torche (2010; véase también Brand y Xie, 2010) replica las conclusiones de Hout, y pasa a mostrar que la tendencia ha continuado desde fines de la década de 1980. Utilizando datos recientes, informa que, en cuanto a estatus ocupacional, ingresos y valor económico total del hijo adulto, el origen social es un factor causal entre aquellos con grado de escuela secundaria o menos, mientras que entre quienes asistieron a la universidad y obtuvieron un grado, el estatus en la adultez se mantiene desconectado del origen social. Así, una vez que uno está en el ámbito de la educación superior, la distribución viene a basarse únicamente en el nivel de educación, tal como mejor rendimiento académico en la universidad, especialidades seleccionadas, y tal vez efectos derivados de la variedad de universidades. Y aunque hay todavía influencia del origen social en la asistencia a la educación superior, con el crecimiento mundial en este sector esta influencia se está debilitando. Dada la creciente homogeneización de la influencia de la escolaridad, no es una sorpresa que esta nueva tendencia en la estratificación se haya replicado 
en Suecia, Francia y Alemania (Vallet, 2004; Breen y Jonsson, 2007; Breen y Luijkx, 2007).

A menudo, el giro hacia la distribución de la educación es atribuido simplemente a una mayor complejidad económica y social, particularmente en la teoría de la reproducción de las clases sociales y la teoría del capital humano, que emplean el mismo modelo tradicional de la educación como institución secundaria. Y sin duda las economías han cambiado, las granjas familiares y las pequeñas empresas declinan, etc., pero lo que a menudo se pierde en todo este cambio social es que reemplazar los antiguos mecanismos de movilidad social de una manera tan rápida y total, como ha hecho la revolución educativa, implica mucho más cambio sociológico que la complejidad económica y social general. Con la excepción tal vez de la formación de aprendices, los mecanismos no educacionales de consecución de estatus arriba mencionados no son conocidos por la mayoría en la sociedad moderna y, sin embargo, antes de la revolución educativa estaban profundamente incrustados en las sociedades y eran considerados maneras totalmente legítimas de clasificar a los individuos en su estatus como adultos, incluyendo las ocupaciones. Pero el ascenso de la educación como el único árbitro de la distribución ha sido tan completo que los anteriores procesos de distribución - sinecura, herencia ocupacional, matrimonio, carisma religioso, adiestramiento en oficios, mecenazgo, casta - aparecen ahora como exóticas reliquias sociales. Aunque en algunas sociedades premodernas la educación formal controlaba el acceso a algunas posiciones de élite, el hecho de que ahora singularmente lo haga así en casi todas las posiciones de estatus habría sido una idea extraña hace apenas cien años. Aunque la distribución educativa parece a muchos contemporáneos una "manera natural" de distribuir, en realidad es una construcción sociológica radical en el fundamento mismo de la sociedad posindustrial.

La cuestión más grande aquí es que estas dos funciones impulsan dinámicamente a la educación como institución a una mayor prominencia en la sociedad posindustrial. Como experiencia transformadora con serias consecuencias sociales, la educación se vuelve cada vez más importante para los individuos y las instituciones sociales por igual. Esto establece el escenario para el amplio impacto de la educación como institución propuesto más arriba. 


\section{Conclusión}

Aunque el público seguirá sobrestimando el potencial de la educación, los profesionales del desarrollo y los analistas de política deben reconsiderar su reacción crítica habitual que, a menudo, conduce a una subestimación del impacto de la revolución educativa. Tanto para los individuos como para otras instituciones sociales, la escolaridad formal generalizada ha desempeñado un papel importante en el desarrollo social en términos de salud y otros sectores relacionados. El abrumador número de asociaciones empíricas entre nivel de educación y actitudes y comportamientos avala este efecto. En lugar de asumir el efecto educación como secundario, o peor, como algo no educacional, sería beneficioso continuar un examen completo de las facetas causales de asistir a la escuela.

El argumento aquí tiene tanto implicaciones para la investigación futura como para el desarrollo social y las políticas. La investigación futura debe incorporar un análisis multidisciplinario de los efectos de la escolaridad en el desarrollo neurológico y las habilidades cognitivas. Demasiado a menudo se asume que el logro escolar es solo resultado de la maduración neurológica anterior, pero hay un creciente cuerpo de evidencia que sugiere que la escolaridad formal tiene un efecto de maduración propio sobre el crecimiento neurológico y cognitivo del individuo (Baker et al., s. f.). Una vez que esta conclusión se hace obvia, se necesitan otras investigaciones para entender exactamente qué hay en el aprendizaje de la lectura, la escritura y el cálculo que mejora el desarrollo cerebral y aumenta las habilidades cognitivas generales tales como el razonamiento y la función ejecutiva, que es tan importante para el pensamiento de orden superior. Así, la literatura sobre esto hasta ahora sugiere que el efecto de maduración de la exposición a la escolaridad dura hasta bien entrada la adolescencia e incluso más tiempo.

Nuevas investigaciones sobre la cognición están mostrando que la emoción y la motivación son un componente fundamental del pensamiento efectivo. La hipótesis escolaridad-cognición-desarrollo social no incluye la evidencia de que la escolaridad puede también aumentar los niveles de autoeficacia y autonomía del individuo para utilizar sus habilidades cognitivas en la resolución de problemas. Esto bien puede resultar una adición importante a la hipótesis desarrollada aquí, y espera investigaciones futuras.

Las implicaciones de política son claras. En primer lugar, las constataciones y el desarrollo de perspectivas aquí formuladas refuerzan aun más los 90 I esfuerzos de gobiernos y organismos de ayuda para difundir la educación 
con un nivel razonable de calidad como un componente y causa principal del desarrollo social y económico futuro de una nación. Después de la seguridad alimentaria y el acceso a servicios de salud, la educación formal es la mejor inversión que puede hacerse en el desarrollo de las poblaciones humanas. Asimismo, si bien se ha hecho mucho para hacer de la inversión en la primera infancia una prioridad, en parte justificada por la noción de que el desarrollo cognitivo se produce mayormente entre los 3 y 5 años de edad, esto no casa con las constataciones aquí expuestas. Y esto conduce a una recomendación de política final. Aunque la expansión de la educación se está produciendo en muchas naciones en desarrollo, muchos jóvenes y adultos jóvenes están severamente no escolarizados. Si la hipótesis escolaridad-cognición-desarrollo social resulta correcta, sugiere una forma de desarrollar intervenciones educativas de corto plazo para jóvenes y adultos que se centren en el desarrollo cognitivo, la lectura y escritura básicas y el cálculo.

Por último, cabe señalar que el argumento desarrollado aquí no pretende implicar que hay solo un efecto unidireccional entre educación y resultados sociales y económicos. En el mundo real hay cualquier número de relaciones sinérgicas entre una población educada y el desarrollo. Por ejemplo, la salud infantil influye de manera importante en la capacidad de ganancia cognitiva en la primera escolaridad, como también la buena nutrición. El punto aquí no es una reducción ingenua de la complejidad de la sociedad, sino más bien hacer hincapié en la centralidad de la educación para el desarrollo, sin lo cual estas sinergias no pueden ocurrir.

Ciertamente, la demanda de educación continuará; no ha disminuido en más de 100 años. Y, por la manera en que la educación se ha convertido tanto en un proceso de transformación como en el camino principal para el estatus social, no hay fin a la vista para la revolución educativa. Este proceso tiene varias consecuencias importantes para las políticas relacionadas con el desarrollo social. En primer lugar, esto explica por qué la demanda de más acceso a la educación seguirá aumentando, como también la demanda de educación de calidad, incluso entre generaciones de padres con bajos niveles de educación propios. En segundo lugar, incluso aunque el nivel de educación promedio aumente dentro de una población, los que tienen más educación tenderán a cosechar beneficios cognitivos junto con ventajas sociales y económicas. 


\section{Referencias}

Baker, D. P. (s. f.). The schooled society: The educational transformation of postindustrial life. Manuscrito enviado para su publicación.

Baker, D. P. (2009). The educational transformation of work: Towards a new synthesis. Journal of Education and Work, 22(3), 163-191.

Baker, D. P., León, J. y Collins, J. M. (2010). Facts, attitudes and health reasoning about HIV and AIDS: Explaining the education effect on condom use among adults in Sub-Saharan Africa. AIDS and Behavior, 15(7), 1319-1327.

Baker, D. P.; León, J.; Smith, E. G.; Collins, J. y Movit, M. (2011). The education effect on population health: A reassessment. Population and Development Review, 37(2), 307-332.

Baker, D. P. y LeTendre, G. K. (2005). National differences, global similarities: World culture and the future of schooling. Stanford: Stanford University Press.

Baker, D. P.; Salinas, D. y Eslinger, P. J. (s. f.). An envisioned bridge: Schooling as a neurocognitive developmental institution. Manuscrito enviado para su publicación.

Blair, C. (2006). How similar are fluid cognition and general intelligence? A developmental neuroscience perspective on fluid cognition as an aspect of human cognitive ability. Behavioral and Brain Sciences, 29, 109-160.

Blair, C.; Gamson, D.; Thorne, S. y Baker, D. P. (2005). Rising mean IQ: Cognitive demand of mathematics education for young children, population exposure to formal schooling, and the neurobiology of the prefrontal cortex. Intelligence, 33(1), 93-106.

Brand, J. E. y Xie, Y. (2010). Who benefits most from college? Evidence for negative selection in heterogeneous economic returns to higher education. American Sociological Review, 75(2), 273-302.

Breen, R. y Jonsson, J. O. (2007). Explaining change in social fluidity: Educational equalization and educational expansion in twentieth-century Sweden. American Journal of Sociology, 112(6), 1775-1810.

Breen, R. y Luijkx, R. (2007). Social mobility and education: A comparative analysis of period and cohort trends in Britain and Germany. En R. P. S. Scherer, G. Otte y M. Gangl (Eds.), Trends and mechanisms in social stratification research (pp. 102-124). Nueva York: Campus. 
Bruine de Bruin, W.; Parker, A. M. y Fischhoff, B. (2007). Individual differences in adult decision-making competence. Journal of Personality and Social Psychology, 92(5), 938-956.

Ceci, S. J. (1991). How much does schooling influence general intelligence and its cognitive components? A reassessment of the evidence. Developmental Psychology, 27(5), 703-722.

Christian, K.; Bachman, H. J. y Morrison, F. J. (2001). Schooling and cognitive development. En R. J. Sternberg y E. Grigorenko (Eds.), Environmental effects on cognitive abilities (pp. 287-335). Mahwah, NJ: Lawrence Erlbaum Associates.

Cleland, J. G. y Van Ginneken, J. K. (1988). Maternal education and child survival in developing countries: The search for pathways of influence. Social Science \& Medicine, 27(12), 1357-1368.

Cole, M. (1996). Cultural psychology: A once and future discipline. Cambridge: The Belknap Press of Harvard University Press.

Drori, G.; Meyer, J. W. y Hwang, H. (2006). Globalization and organization: World society and organizational change. Oxford: Oxford University Press.

Duncan, J.; Burgess, P. y Emslie, H. (1995). Fluid intelligence after frontal lesions. Neuropsychologia, 33, 261-268.

Eslinger, P. J.; Blair, C.; Wang, J.; Lipovsky, B.; Realmuto, J.; Baker, D. y Yang, Q. $X$. (2009). Developmental shifts in $\mathrm{fMRI}$ activations during visuospatial relational reasoning. Brain and Cognition, 69(1), 1-10.

Eslinger, P. J.; Flaherty-Craig, C. V. y Benton, A. L. (2004). Developmental outcomes after early prefrontal cortex damage. Brain and Cognition, 55(1), 84-103.

Frank, D. y Gabler, J. (2006). Reconstructing the university: Worldwide shifts in academia in the 20th century. Stanford, CA: Stanford University Press.

Hobcraft, J. (1993). Women's education, child welfare and child survival: A review of the evidence. Health Transition Review, 3(2), 159-175.

Hout, M. (1984). Status, autonomy, and training in occupational mobility. The American Journal of Sociology, 89(6), 1379-1409.

Hout, M. (1988). More universalism, less structural mobility: The american occupational structure in the 1980s. The American Journal of Sociology, 93(6), 1358-1400. 
Kamens, D. (2009). The expanding polity: Theorizing the links between expanded higher education and the new politics of the post-1970s. American Journal of Education, 116(1), 99-124.

Kamens, D. H.; Meyer, J. W. y Benavot, A. (1996). Worldwide patterns in academic secondary education curricula. Comparative Education Review, 40(2), 116-138.

Luria, A. R. (1976). Cognitive development: Its cultural and social foundations. Cambridge: Harvard University Press.

Martinez, M. E. (2000). Education as the cultivation of intelligence. Nueva Jersey: Erlbaum Associates.

Meyer, J. W. (1977). The effects of education as an institution. American Journal of Sociology, 83(1), 55-77.

Parsons, T. (1971). Higher education as a theoretical focus. En H. Turk y R. L. Simpson (Eds.), Institutions and social exchange: The sociologies of Talcott Parsons and George C. Homans (pp. 233-252). Indianápolis: Bobbs-Merill.

Peters, E.; Baker, D. P.; Dieckmann, N. F.; León, J. y Collins, J. (2010). Explaining the effect of education on health. Psychological Science, 21(10), 13691376 .

Peters, E.; Västfjäll, D.; Slovic, P.; Mertz, C.; Mazzocco, K. y Dickert, S. (2006). Numeracy and decision making. Psychological Science, 17(5), 407-413.

Schaub, M. (2010). Parenting for cognitive development from 1950 to 2000: The institutionalization of mass education and the social construction of parenting in the United States. Sociology of Education, 83(1), 46-66.

Schwadel, P. (2003). The persistence of religion: The effects of education on american christianity (tesis doctoral no publicada). Pennsylvania State University, University Park, PA.

Shallice, T. y Burgess, P. (1991). Higher-order cognitive impairments and frontal lobe lesions in man. En H. M. Eisenberg y A. L. Benton (Eds.), Frontal lobe function and dysfunction (pp. 125-138). New York: Oxford University Press.

Smith, C. (2003). The secular revolution: Power, interests, and conflict in the secularization of American public life. Berkeley: University of California Press.

Stevenson, H. W.; Chen, C. y Booth, J. (1990). Influences of schooling and urbanrural residence on gender differences in cognitive abilities and academic achievement. Sex Roles, 23(9), 535-551. 
Stevenson, H. W.; Parker, T.; Wilkinson, A.; Bonnevaux, B.; Gonzalez, M. y Greenfield, P. M. (1978). Schooling, environment, and cognitive development: A cross-cultural study. Monographs of the Society for Research in Child Development, 43, 1-92.

Stinchcombe, A. y March, J. (1965). Handbook of organizations. Chicago: Rand McNally.

Torche, F. (2010). Is a college degree still the great equalizer? Intergenerational mobility across levels of schooling in the U.S. (Working Paper). Nueva York: New York University Center for Advanced Social Science Research.

UNESCO. (2002). Education for all: Is the world on track? (EFA Monitoring Report). París: UNESCO.

Vallet, L.-A. (2004). Change in intergenerational class mobility in France from the 1970s to the 1990s and its explanation: An analysis following the CASMIN approach. En R. Breen (Ed.), Social mobility in Europe (pp. 115147). Oxford: Oxford University Press.

Young, M. (2008). Bringing knowledge back in: From social constructivism to social realism in the sociology of education. London: Routledge.

Manuscrito recibido: 01 de diciembre, 2010

Revisado: 17 de agosto, 2011

Aceptado: 29 de agosto, 2011 